\title{
Relationships Between Self-awareness and Clinical Diagnostic Findings of Abnormal Foot Arch Height in Koreans
}

\author{
Youngsuk Son, $\mathrm{MD}^{1}$, Hyo Jeong Kang, PT, $\mathrm{MS}^{2}$, Yun-Mi Song, MD, $\mathrm{PhD}^{3}$, Ji Hye Hwang, MD, PhD ${ }^{1}$
}

\footnotetext{
${ }^{1}$ Department of Physical and Rehabilitation Medicine, Samsung Medical Center, Sungkyunkwan University School of Medicine, Seoul; ${ }^{2}$ Department of Physical Therapy, Graduate School of Sahmyook University, Seoul;

${ }^{3}$ Department of Family Medicine, Samsung Medical Center, Sungkyunkwan University School of Medicine, Seoul, Korea
}

\begin{abstract}
Objective To see how people think about their own feet, and evaluate whether there are correlations among selfawareness of the participants and clinical examination findings.

Methods Adult twins and their families who participated in the Healthy Twin study from May 2008 to April 2010 were recruited. Participants were asked whether they thought their feet were normal, flat, or cavus. The lateral talometatarsal angles were measured on foot X-rays to determine the foot arch height. Using the podoscopic footprints taken with the podobaroscope, the Staheli arch index was also measured. Kappa statistics were used to calculate degree of agreement among the three measurement methods.

Results Self-awareness and radiographic findings were significantly different (Pearson chi-square test, $\mathrm{p}=0.000$ ) and only slightly agreed (kappa measure of agreement $=0.136, p=0.000$ ). Self-awareness and podoscopy results revealed a significant difference (Pearson chi-square test, $\mathrm{p}=0.000$ ), with only slight agreement (kappa measure of agreement $=0.072, \mathrm{p}=0.000$ ).

Conclusion There is significant disagreement between patients' perception of their feet and actual test results. Many people may have an incorrect assumption about their own foot conditions that may be reflected in improper management. Dissemination of accurate information about foot disorders by foot clinicians would be helpful.
\end{abstract}

Keywords Flatfoot, Talipes cavus

\section{INTRODUCTION}

Foot types differ according to foot shape and foot arch height. Flatfoot and cavus foot are common but complex foot deformities. The flatfoot deformity is characterized by a combination of a collapse of the medial longitudinal

Received March 22, 2017; Accepted May 30, 2017

Corresponding author: Ji Hye Hwang

Department of Physical and Rehabilitation Medicine, Samsung Medical Center, Sungkyunkwan University School of Medicine, 81 Irwon-ro, Gangnamgu, Seoul 06351, Korea. Tel: +82-2-3410-2816, Fax: +82-2-3410-0052, E-mail: hwanglee@skku.edu

ORCID: Youngsuk Son (http://orcid.org/0000-0003-1328-4631); Hyo Jeong Kang (http://orcid.org/0000-0003-3433-4845); Yun-Mi Song (http://orcid. org/0000-0001-9232-5563); Ji Hye Hwang (http://orcid.org/0000-0002-8176-3354).

(c) This is an open-access article distributed under the terms of the Creative Commons Attribution Non-Commercial License (http://creativecommons.org/ licenses/by-nc/4.0) which permits unrestricted noncommercial use, distribution, and reproduction in any medium, provided the original work is properly cited. Copyright (c) 2017 by Korean Academy of Rehabilitation Medicine 
arch, foot abduction at the talonavicular joint, and hindfoot valgus $[1,2]$. The cavus foot deformity is the opposite. Either deformity may be a predisposing factor for injuries in the lower extremity [3-5].

In Korea, the concept of foot deformity is especially well-known in the general population, especially flatfoot. Much of the recognition is because flatfoot is an important physical examination feature for military qualification in South Korea. The qualification examination for the Korean military includes plantar measurement, since flatfoot may result in various podiatric symptoms or functional impairment in soldiers who are already prone to pain or injuries in lower extremities due to excessive marching or weight-bearing activities.

While the concept of this disorder is widely familiar, the diagnosis of this condition is controversial. The reported prevalence of flatfoot varies and ranges from approximately $0.6 \%-77.9 \%$ [6]. One study reported prevalence of $19.0 \%$ [7] and another reported a prevalence of $23 \%$ including asymptomatic flatfoot [8]. The marked variation in prevalence rates may be related to ethnic or behavioral differences among study populations and different diagnostic criteria.

Diagnosis of flatfoot can be made by physical examination, radiographic assessment, and footprint analysis. Correlations between these diagnostic tools have been described [9-17]. Despite the numerous studies concerning the clinical relevance of the different diagnostic tools, no study has addressed patients' self-awareness of their feet or the correlation of the self-perception with objective clinical findings.

This study prospectively collected data of participants' self-awareness about their foot shape, as well as radiographic findings and podoscopic findings. The aim was to clarify how people perceive their foot conditions and evaluate whether there are correlations with clinical findings.

\section{MATERIALS AND METHODS}

\section{Subjects}

Adult twins and their families who participated in the Healthy Twin study from May 2008 to April 2010 were recruited. Participants received full medical examinations and completed detailed questionnaires about lifestyle and epidemiologic information. Details on the study de- sign and protocols have been previously published [18]. Physical examinations by a well-trained physical therapist and radiographic measurements of feet were done at the Samsung Medical Center. All participants provided written informed consent. The study protocol was approved by the Institutional Review Board (IRB No. SMC 2005-08-113) at Board at Samsung Medical Center and Seoul National University of Public Health.

\section{Questionnaires}

Self-awareness about foot deformities, shoe type, shoe wearing habit, pain, transportation, and etiologic factors were questioned. If participants suffered foot pain, they were queried on the location of pain (forefoot, midfoot, and hindfoot) and the severity of pain according to a numeric pain rating scale (NRS). To evaluate self-awareness about foot deformities, participants were asked whether they thought their feet were normal, flat, or cavus.

\section{Radiographic assessment}

The assessment included lateral and anteroposterior weight-bearing views of the foot. Lateral weight-bearing views were used. The lateral talometatarsal angle (angle between the talar axis and the axis of the first metatarsal) was measured as previously described [16] (Fig. 1). The talometatarsal angle is considered an accurate radiographic identifier of patients with symptomatic, adult flatfoot $[14,15,19]$. This angle is used as a diagnostic tool for flatfoot in Korean military qualification examinations. The normal accepted range of this measurement is $-4^{\circ}$ to $4^{\circ}[16,19]$. Patients with angles above $4^{\circ}$ were considered to have pes planus, and those with angles below $-4^{\circ}$ were

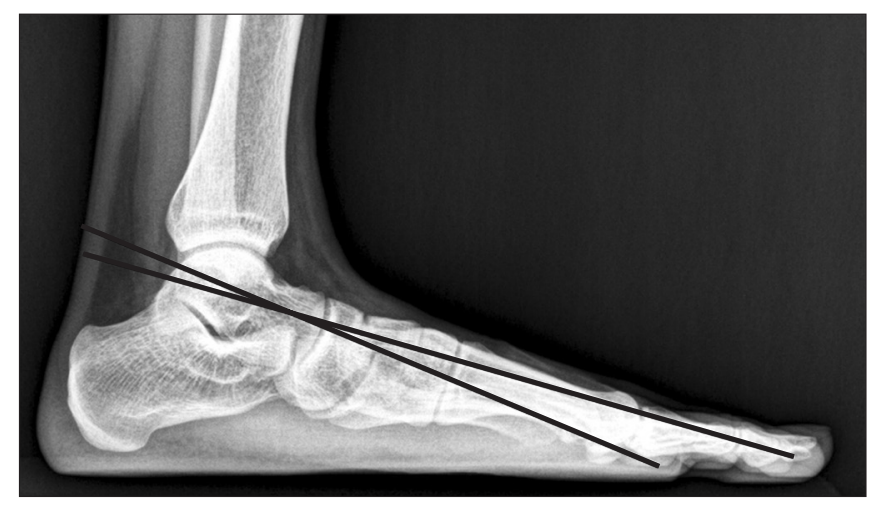

Fig. 1. Measurement of the talometatarsal angle. 
considered to have pes cavus.

\section{Podoscopic assessment}

Podoscopic footprints were taken with the participant standing in a weight-bearing stance on the podobaroscope (Guy Capron, Paris, France). The Staheli arch index was measured. This index is the ratio between the widest region of the midfoot and the heel [20] (Fig. 2). Based

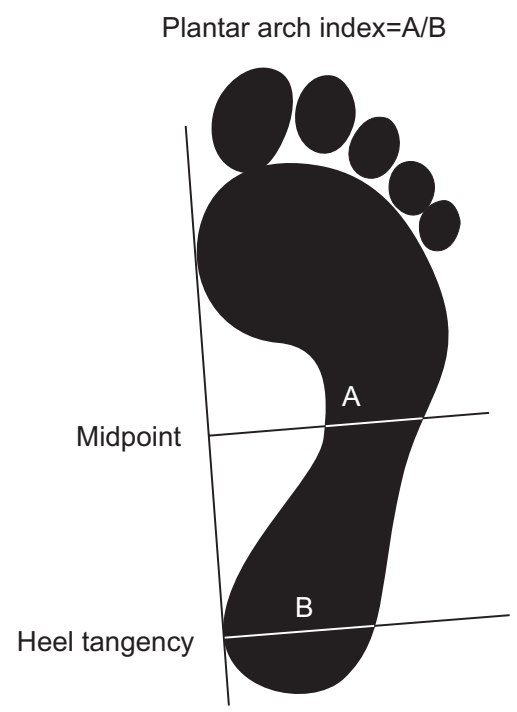

Fig. 2. Measurement of the Staheli arch index. on the Staheli arch index, participants were categorized on a scale from 1 to $5: 1=$ flat $(>1.2) ; 2=$ low arch (0.9-1.2); $3=$ normal $(0.5-0.9) ; 4=$ high arch $(0.2-0.5)$; and $5=$ cavus $(<0.2)[21]$.

\section{Statistical analyses}

Kappa statistics were used to calculate degree of agreement among the three measurement methods. The level of significance was set at 0.05 . Analyses were executed using SAS ver. 9.4 (SAS Institute, Cary, NC, USA).

\section{RESULTS}

A total of 1,277 individuals participated in this study. They comprised 500 males and 777 females. The mean age was 44.0 years. Of the 1,277 individuals, 488 individuals showing discordance in radiographic or podoscopic findings were excluded. The remaining 789 participants comprised 320 males and 469 females, with a mean age of 43.90 years. Of the $789,712(90.24 \%)$ perceived their feet as normal, 69 (8.75\%) regarded their feet as flat, and $8(1.01 \%)$ perceived their feet as cavus. According to the talometatarsal angle, 467 (59.19\%) were diagnosed as normal, $174(22.05 \%)$ as flatfeet, and $148(18.76 \%)$ as cavus feet. Podoscope examination diagnosed 430 (54.50\%) as normal, $54(6.84 \%)$ as flatfeet, and $305(38.66 \%)$ as

Table 1. General characteristics of study participants with symmetric radiographic and podoscopic results $(\mathrm{n}=789)$

\begin{tabular}{|lccc|}
\hline Characteristic & Total $(\mathbf{n}=\mathbf{7 8 9})$ & Male $(\mathbf{n}=\mathbf{3 2 0})$ & Female $(\mathbf{n}=\mathbf{4 6 9})$ \\
\hline Age $(\mathrm{yr})$ & $43.90 \pm 13.67$ & $44.21 \pm 14.68$ & $43.68 \pm 12.95$ \\
\hline Height $(\mathrm{cm})$ & $162.62 \pm 8.84$ & $170.51 \pm 6.52$ & $157.23 \pm 5.56$ \\
\hline Body weight $(\mathrm{kg})$ & $63.30 \pm 11.93$ & $71.69 \pm 11.28$ & $57.57 \pm 8.48$ \\
\hline BMI $\left(\mathrm{kg} / \mathrm{m}^{2}\right)$ & $23.83 \pm 3.31$ & $24.58 \pm 3.07$ & $23.31 \pm 3.37$ \\
\hline Self-awareness & & & \\
\hline Normal & $712(90.24)$ & $294(91.88)$ & $418(89.13)$ \\
\hline Flat foot & $69(8.75)$ & $25(7.81)$ & $74(9.38)$ \\
\hline Cavus foot & $8(1.01)$ & $1(0.31)$ & $7.49)$ \\
\hline Talometatarsal angle & & & $276(58.85)$ \\
\hline Normal & $467(59.19)$ & $34(10.03)$ & $73(15.57)$ \\
\hline Flat foot & $174(22.05)$ & $43(12.68)$ & $242(51.60)$ \\
\hline Cavus foot & $148(18.76)$ & & $33(7.04)$ \\
\hline Podoscopy & & $188(58.75)$ & $194(41.36)$ \\
\hline Normal & $430(54.50)$ & $21(6.56)$ & \\
\hline Flat foot & $54(6.84)$ & $111(34.69)$ & \\
\hline Cavus foot & $305(38.66)$ & & \\
\hline
\end{tabular}

Values are presented as mean \pm standard deviation or number $(\%)$. 
cavus feet. General characteristics of the participants are provided in Table 1.

Differences between participants' self-awareness about their feet and the clinical diagnosis based on talometatarsal angle measurements were compared (Table 2). Of the 69 participants who thought their feet were flat, $41(59.42 \%)$ were actually diagnosed as flatfeet, and 19 (27.54\%) were normal according to radiographic findings. Of the 174 participants diagnosed as flatfeet by radiologic findings, only $41(23.56 \%)$ thought their feet were flat, with $132(75.86 \%)$ perceiving their feet to be normal. Of the 8 participants who thought their feet were cavus, $2(25.00 \%)$ were actually diagnosed as cavus feet and $5(62.50 \%)$ showed normal talometatarsal angles. Of the 148 participants diagnosed as cavus feet according to their talometatarsal angle, 137 (92.57\%) perceived their feet as normal and only $2(1.35 \%)$ regarded their feet as caval. The two findings were significantly different (Pearson chi-square test, $\mathrm{p}=0.000$ ), with only slight agreement (kappa measure of agreement $=0.136, \mathrm{p}=0.000$ ).

We also compared self-awareness with diagnosis according to the podoscopic findings (Table 3). Of the 69 individuals who answered that they had flatfeet, only 27 (39.13\%) were actually diagnosed as flatfeet, with 35 (50.72\%) having normal feet. Fifty-four participants were diagnosed as flatfeet according to podoscopy results; 27 (50.00\%) perceived their feet as normal and the other 27 $(50.00 \%)$ thought they had flatfeet. Of the 8 participants who regarded their feet as caval, $5(62.50 \%)$ actually had cavus feet and $3(37.50 \%)$ were normal on podoscopy. Of the 305 participants with cavus feet on podoscopy, only $5(1.65 \%)$ thought their feet were cavus feet. The

Table 2. Self-awareness versus talometatarsal angle

\begin{tabular}{|ccccc}
\hline \multirow{2}{*}{ Talometatarsal angle } & \multicolumn{4}{c}{ Self-awareness } \\
\cline { 2 - 5 } & Normal & Flat foot & Cavus foot & Total \\
\hline Normal & $443(62.22)$ & $19(27.54)$ & $5(62.50)$ & 467 \\
Flat foot & $132(18.54)$ & $41(59.42)$ & $1(12.50)$ & 174 \\
Cavus foot & $137(19.24)$ & $9(13.04)$ & $2(25.00)$ & 148 \\
Total & 712 & 69 & 8 & 789 \\
\hline
\end{tabular}

Values are presented as number (\%).

Pearson chi-square $=55.130, \mathrm{df}=4, \mathrm{p}=0.000$.

Table 3. Self-awareness versus podoscopy results

\begin{tabular}{|lcccr}
\hline \multirow{2}{*}{ Podoscopy } & \multicolumn{4}{c}{ Self-awareness } \\
\cline { 2 - 5 } & Normal & Flat foot & Cavus foot & Total \\
\hline Normal & $392(55.06)$ & $35(50.72)$ & $3(37.50)$ & 430 \\
Flat foot & $27(3.79)$ & $27(39.13)$ & $0(0)$ & 54 \\
Cavus foot & $293(41.15)$ & $7(10.14)$ & $5(62.50)$ & 305 \\
\hline Total & 712 & 69 & 8 & 789 \\
\hline
\end{tabular}

Values are presented as number (\%).

Pearson chi-square $=111.862, \mathrm{df}=4, \mathrm{p}=0.000$.

Table 4. Talometatarsal angle $\left({ }^{\circ}\right)$ of flatfoot group according to self-awareness or podoscopy

\begin{tabular}{ccccccc}
\hline $\begin{array}{c}\text { Talometatarsal } \\
\text { angle }\end{array}$ & $\begin{array}{c}\text { Self-awareness: } \\
\text { Flat foot } \\
(\mathbf{n = 6 9 )}\end{array}$ & $\begin{array}{c}\text { Podoscopy: } \\
\text { Flat foot } \\
(\mathbf{n = 5 4 )}\end{array}$ & $\begin{array}{c}\text { Self-awareness: } \\
\text { Normal } \\
(\mathbf{n = 7 1 2})\end{array}$ & $\begin{array}{c}\text { Podoscopy: } \\
\text { Normal } \\
(\mathbf{n = 4 3 0})\end{array}$ & $\begin{array}{c}\text { Self-awareness: } \\
\text { Cavus foot } \\
(\mathbf{n = 8})\end{array}$ & $\begin{array}{c}\text { Podoscopy: } \\
\text { Cavus foot } \\
(\mathbf{n}=\mathbf{3 0 5})\end{array}$ \\
\hline Total & $-4.77 \pm 8.04$ & $-8.50 \pm 7.40$ & $-0.13 \pm 5.55$ & $-1.33 \pm 5.24$ & $0.94 \pm 4.88$ & $2.02 \pm 4.96$ \\
Right foot & $-4.58 \pm 7.58$ & $-8.22 \pm 7.05$ & $0.00 \pm 5.59$ & $-1.25 \pm 5.24$ & $0.50 \pm 5.15$ & $2.19 \pm 4.99$ \\
\hline Left foot & $-4.96 \pm 8.54$ & $-8.76 \pm 7.79$ & $-0.26 \pm 5.51$ & $-1.4 \pm 5.25$ & $1.38 \pm 4.90$ & $1.84 \pm 4.92$ \\
\hline
\end{tabular}

Values are presented as mean \pm standard deviation. 


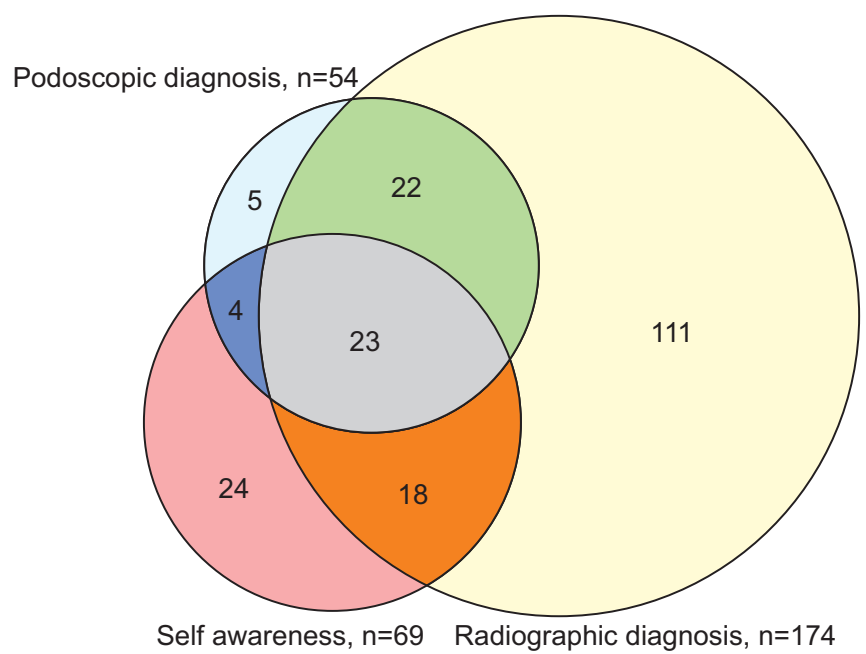

Fig. 3. Distribution of flatfoot according to self-awareness, radiographic findings, and podoscopic findings.

overwhelming majority $(\mathrm{n}=293,96.07 \%)$ thought their feet were normal. These results showed a significant difference (Pearson chi-square test, $\mathrm{p}=0.000$ ), with only slight agreement (kappa measure of agreement $=0.072$, $\mathrm{p}=0.000$ ).

The mean talometatarsal angle of participants who perceived their feet as being flatfeet was $-4.77^{\circ} \pm 8.04^{\circ}$. Those actually diagnosed as flatfeet according to podoscopy showed a mean talometatarsal angle of $-8.50^{\circ} \pm 7.40^{\circ}$ (Table 4).

Fig. 3 shows the distribution of the number of individuals who either thought they had flatfeet or who presented test findings consistent with flatfeet. It is obvious that there were more people with inconsistent findings than consistent results.

\section{DISCUSSION}

Flatfoot is a common disorder, and is likely the most recognized foot deformity in the general Korean populations. Most healthy Korean males in their 20s are mandatorily recruited for military service. Military training consists of vigorous weight-bearing physical activities including marching and mountain climbing. This physical burden likely heightens the vulnerability of soldiers to musculoskeletal pain or injuries, especially in the lower extremities. In one study, of 287 soldiers treated at a military hospital, $74.9 \%$ involved lower extremity injuries [22]. Since flatfoot may increase the risk of lower extremity in- juries and pain, the Korean military qualification examination includes plantar arch assessment.

The widespread recognition of flatfoot made us wonder how Koreans perceive their plantar arches. Many studies have addressed the relationships among physical examination findings, radiographic parameters, and podoscopic findings. But, no study has addressed selfawareness. Among the various radiographic examination tools, we chose the talometatarsal angle for the present study. Previous studies concluded that the lateral talometatarsal angle is the best radiographic angle to quantify the height of the medial longitudinal arch $[14,19,23]$. As a podoscopic assessment, we used Staheli arch index, which correlates with the radiographic parameters and is simple to use $[20,21,24]$.

There was significant disagreement between patients' perception of their own feet and actual test results. Several explanations can be offered. One possibility is that current diagnostic examinations may be insufficient for diagnosis of flatfoot. These examinations may not accurately demonstrate patients' actual symptoms and thus show poor correlations. However, considering previous demonstrated correlations between clinical symptoms and these diagnostic tools $[14,15,19]$, this explanation needs further study.

Another possibility is that the Korean general population may have an incorrect understanding about the concept of flatfoot. While flatfoot is a widely recognized term, it may be that few Koreans actually fully understand the pathophysiology and clinical presentations of the disorder. Especially, people are likely to think they have some pathological conditions when they actually have discomfort. This may lead people with asymptomatic flatfeet to think their feet are normal. On the other hand, there may be various clinical conditions causing foot symptoms. It could be easy for people with any foot symptoms to regard themselves as having flatfeet, as the disorder is so familiar to them while other disorders are not as familiar.

Either way, these results imply that many people may have wrong assumptions about their own foot conditions and may not properly manage their feet. Therefore it would be ideal to introduce to the population the exact definition, physiology and clinical symptoms of the flatfoot and other disorders that may cause foot symptoms as well. It would be helpful if there are ways to popularize such information via various media. An opportunity is 
during visits to foot clinics. This would also allow proper foot management before hasty assumptions are made. A good first step would be the dissemination of accurate information to clients by foot clinicians.

\section{CONFLICT OF INTEREST}

No potential conflict of interest relevant to this article was reported.

\section{REFERENCES}

1. Arunakul M, Amendola A, Gao Y, Goetz JE, Femino JE, Phisitkul P. Tripod index: a new radiographic parameter assessing foot alignment. Foot Ankle Int 2013;34: 1411-20.

2. Dyal CM, Feder J, Deland JT, Thompson FM. Pes planus in patients with posterior tibial tendon insufficiency: asymptomatic versus symptomatic foot. Foot Ankle Int 1997;18:85-8.

3. Clement DB, Taunton JE. A guide to the prevention of running injuries. Can Fam Physician 1980;26:543-8.

4. Lysholm J, Wiklander J. Injuries in runners. Am J Sports Med 1987;15:168-71.

5. Williams DS, McClay IS. Measurements used to characterize the foot and the medial longitudinal arch: reliability and validity. Phys Ther 2000;80:864-71.

6. Halabchi F, Mazaheri R, Mirshahi M, Abbasian L. Pediatric flexible flatfoot; clinical aspects and algorithmic approach. Iran J Pediatr 2013;23:247-60.

7. Dunn JE, Link CL, Felson DT, Crincoli MG, Keysor JJ, McKinlay JB. Prevalence of foot and ankle conditions in a multiethnic community sample of older adults. Am J Epidemiol 2004;159:491-8.

8. Harris RI, Beath T. Hypermobile flat-foot with short tendo achillis. J Bone Joint Surg Am 1948;30A:116-40.

9. Harris EJ, Vanore JV, Thomas JL, Kravitz SR, Mendelson SA, Mendicino RW, et al. Diagnosis and treatment of pediatric flatfoot. J Foot Ankle Surg 2004;43:341-73.

10. Menz HB, Fotoohabadi MR, Wee E, Spink MJ. Visual categorisation of the arch index: a simplified measure of foot posture in older people. J Foot Ankle Res 2012;5:10.

11. Lee JS, Kim KB, Jeong JO, Kwon NY, Jeong SM. Correlation of foot posture index with plantar pressure and radiographic measurements in pediatric flatfoot. Ann
Rehabil Med 2015;39:10-7.

12. Lee TH, Chay SW, Kim HJ. Diagnosis of flatfoot deformity. J Korean Foot Ankle Soc 2016;20:1-5.

13. Murley GS, Menz HB, Landorf KB. A protocol for classifying normal- and flat-arched foot posture for research studies using clinical and radiographic measurements. J Foot Ankle Res 2009;2:22.

14. Younger AS, Sawatzky B, Dryden P. Radiographic assessment of adult flatfoot. Foot Ankle Int 2005;26:8205.

15. Kim SB, Yoon K, Park HS, Kwak H, Ha NJ, Park JS. Radiologic measurement of flatfoot. J Korean Acad Rehabil Med 2000;24:995-1001.

16. Gould N. Graphing the adult foot and ankle. Foot Ankle 1982;2:213-9.

17. Pita-Fernandez S, Gonzalez-Martin C, Seoane-Pillado T, Lopez-Calvino B, Pertega-Diaz S, Gil-Guillen V. Validity of footprint analysis to determine flatfoot using clinical diagnosis as the gold standard in a random sample aged 40 years and older. J Epidemiol 2015;25: 148-54.

18. Sung J, Cho SI, Lee K, Ha M, Choi EY, Choi JS, et al. Healthy Twin: a twin-family study of Korea: protocols and current status. Twin Res Hum Genet 2006;9:8448.

19. Coughlin MJ, Kaz A. Correlation of Harris mats, physical exam, pictures, and radiographic measurements in adult flatfoot deformity. Foot Ankle Int 2009;30:60412.

20. Staheli LT, Chew DE, Corbett M. The longitudinal arch. A survey of eight hundred and eighty-two feet in normal children and adults. J Bone Joint Surg Am 1987;69:426-8.

21. Chuckpaiwong B, Nunley JA 2nd, Queen RM. Correlation between static foot type measurements and clinical assessments. Foot Ankle Int 2009;30:205-12.

22. Kim JS, Lee YS. Causal relationship between military activities and musculoskeletal injuries. J Korea Inst Mil Sci Technol 2008;11:142-7.

23. Pedowitz WJ, Kovatis P. Flatfoot in the Adult. J Am Acad Orthop Surg 1995;3:293-302.

24. Kanatli U, Gozil R, Besli K, Yetkin H, Bolukbasi S. The relationship between the hindfoot angle and the medial longitudinal arch of the foot. Foot Ankle Int 2006; 27:623-7. 